International Journal of Pure and Applied Mathematics

Volume 89 No. $2 \quad$ 2013, 173-201

ISSN: 1311-8080 (printed version); ISSN: 1314-3395 (on-line version)

url: http://www.ijpam.eu

doi: http://dx.doi.org/10.12732/ijpam.v89i2.5

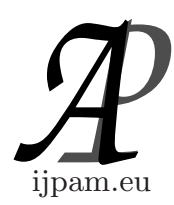

\title{
AN A-CONTRARIO APPROACH FOR OBJECT DETECTION IN VIDEO SEQUENCE
}

\author{
Moez Ammar ${ }^{1}$, Sylvie Le Hégarat-Mascle ${ }^{2} \S$, \\ ${ }^{1,2}$ Marius Vasiliu, and Roger Reynaud \\ University Paris-Sud \\ Orsay, 91405, FRANCE
}

\begin{abstract}
This study focuses on the problem of object detection in video sequences. In order to free from the modeling of the objects that may be variable and numerous, we choose an a-contrario approach, based on the modeling of the absence of any object of interest in the scene (noise model) and the images are analyzed in order to detect the events salient relative to the used noise model. To free from the a-priori parameters, we define a non-monotonic measure that is a Number of False Alarms (NFA) and that allows quantifying the 'exceptional' feature of an observation relatively to the noise model. With such an approach the solution is determined as the argument minimizing the NFA criterion.

Considering an image segmentation problem, the proposed noise model has two, namely pixel and window, levels in order to take into account both radiometric and spatial noise feature. The same algorithm is then used to detect either the changes relative to a background, or in the case of an embedded camera, the objects having their own motion, or the objects protruding relatively to the road plane, so that applications are related to video surveillance and Advanced Driver Assistance Systems.
\end{abstract}

AMS Subject Classification: 68U10, 62M40

Key Words: a-contrario decision, object detection, video

Received: July 26, 2013

(C) 2013 Academic Publications, Ltd. url: www.acadpubl.eu

${ }^{\S}$ Correspondence author 


\section{Introduction}

The problem of detection is all the more difficult as the objects or events to detect are multiple, having variable forms and poorly known. In most practical applications, it will be generally simpler to model the noise (in unstructured data) than to list the various forms of the objects. Thus, instead of specifying the a priori structures or sought objects (events), the a-contrario approaches use a model, called 'naive' model, which represents the absence of structures in the data. A phenomenon (e.g. alignment, change) will be detected as a very unlikely (contradictory) realization under the assumption of naive model, or in other words as 'too structured' (according to the Helmholtz principle, Any structure too regular to appear 'by chance' attracts the attention and becomes a perception).

In order to measure the degree of significance of a 'too structured' observation, [4] introduces a measure directly related to the number of occurrences associated to this observation. If this number is large, the significance is low. The approach allows gathering all the detection parameters (thresholds, etc.) under a single criterion: the expected number of false alarms (NFA). A-contrario approaches using NFA criterion have been applied to various problems of detection of structures in images [6]. Let us quote, in a non-exhaustive way, the alignment of points [4], the contours [5], the vanishing points [1], the line segments [20], the matching points and epipolar geometry measure [15], the matching of local descriptors [17], pattern recognition [2], or change detection (on remote sensing images [18] and in video scenes [9]).

Considering the application of detection of 'unexpected' objects or change in an image, and relatively to mentioned works, we focus on the following key points. First, the considered images are gray-level images whereas in the most of the cited works, e.g. [7], NFA measures were proposed for binary images. Second, in order to free from the use of some threshold parameters, the NFA criterion should present a minimum, whereas in several cited works, e.g. [9], a final thresholding on NFA values is used. Third, both radiometric and spatial features have to be considered in the object detection, whereas cited works only consider one level, either pixel level or window level.

In image processing, the most of the approaches for change detection are based on the interpretation of the image difference between a current (or foreground) image and a background image representing the absence of change or object of interest. Then, numerous works focus on the updating the background image. Indeed, the actual scenes contain structures that vary in terms of static objects (e.g. parked cars) or in acquiring conditions (e.g. light, clouds). Then, 
the Sigma-Delta filter [14] (to give an example) provides an extremely simple and relatively robust way to update the background, through the mean and the median value around the mean.

When the scene is subject to pseudo-periodic variations (e.g. movements of the branches in the trees due to wind), [12] propose a representation of the background image from a dictionary, called CodeBook. Updating of the available backgrounds (included in the CodeBook) is performed to manage non-periodic changes. Including an extra cost in computation time, this model allows managing the cases where in some areas the background cannot be modeled by a single value. Having estimated the background (either mono or multilayer), a test determines if the observed value is significantly away from the background. This test is usually probabilistic (e.g. assuming Gaussian mixture $[22,19,3,16]$ ). Now, when methods work at pixel level, they are prone to errors, and a way to make them more robust is to extend them at window level. Anyway, a main limitation of these approaches remains then the existence of some parameters that should be a priori known or learned and the existence of a final decision threshold.

In this study we focus on the detection of 'unexpected' objects, where the term 'unexpected' refers to something that deviates from a scene model. For instance there will be an appearance (or disappearance) of an object or a movement of an object relative to the known scene. Objects will be detected as innovation relatively to a scene model, defined according to the application, for instance as a background image, as a previous image in the sequence or as a 'default' scene (we will give examples further).

In terms of application, focusing on the case where the object is new, i.e. not considering the tracking problem, three main features make our problem all the more complex:

1. the input data are 'grayscale' (scalar values) images with possibly missing values (typically, the considered grayscale values may be standard radiometric values or disparity values),

2. the acquisition conditions are highly variable (outdoor),

3. the camera may be embedded (and thus in move).

The remainder of the article is as follows. In Section 2, we focus on the acontrario modeling and we present the proposed approach in the case of a static camera. The detection is performed without using any threshold parameter. In Section 3, we show the genericity of the method applying it to various input 
data (observation and model), in the more general case of a camera with egomotion. Section 4 summarizes our main conclusions and give some perspectives for this work.

\section{Proposed Approach}

Assuming that the objects are large relative to the pixel size, they include several pixels. Thus, in the absence of any assumption about their shape, the objects will be detected as groups of pixels that satisfy a specific detection function at the pixel level.

Then, the proposed algorithm includes two steps: first a function detecting the changes at pixel level, and secondly a function detecting the changes at window level. For this presentation of the method, let us first assume a static camera and let us focus on video surveillance examples. The extension to the case of a moving camera is presented in the next section.

\subsection{Level 1: Detection of Change Points in the Image}

The detection at pixel level is based on [18], with two main differences: in our case, the resolution is high (objects are greater than pixel size) and the detection is not based on a comparison with a classification but with a grayscale image (e.g. image background or image derived from a 3D scene model).

The proposed detection at pixel level is based on the comparison of the observed image at the instant $t$, the innovation, noted $I_{t}$, and a model at $t$, noted $\tilde{B}_{t}$. In the case of a static camera, $\tilde{B}_{t}$ is a background image. Let $\mathcal{D}_{t}$ be the domain of defined pixels, i.e. pixels having a value assumed to be reliable. Examples of pixels not belonging to $\mathcal{D}_{t}$ are: blind, hot pixels, or pixels with undefined value such as in disparity maps. In this section, all calculations are carried out for a given time index $t$. Thus, to simplify the notation the index $t$ is removed in the following of this section: we note $I=I_{t}, \tilde{B}=\tilde{B}_{t}$ and $\mathcal{D}=\mathcal{D}_{t}$. Let us also note $\overline{\mathcal{D}}$ the unknown pixel domain, defined as the complementary of $\mathcal{D}$ relatively to the whole image domain.

Let us note $\Delta$ the difference image: $\forall s \in \mathcal{D}, \Delta(s)=I(s)-\tilde{B}(s)$. Classic thresholding is performed on the values $|\Delta(s)|$ or $\Delta^{2}(s)$ to determine the value of pixels surprisingly high. But the choice of the threshold is generally very difficult and not robust. In order to determine the threshold automatically, the significance of the pixels of the image $\Delta$ is measured with respect to the naive model $H_{1}$, given by Definition 1 . 
Definition 1 (naive model $H_{1}$ ). The image $\Delta$ is a random field of $|\mathcal{D}|$ independent centered Gaussian variables $\mathcal{N}\left(0, \sigma^{2}\right)$ with $\sigma$ the corresponding standard deviation image completely unstructured and $|\mathcal{D}|$ the cardinality of the domain $\mathcal{D}$ of defined pixels.

Let $\Delta^{2}$ be the image such that $\Delta^{2}(s)=[\Delta(s)]^{2}$. The residual error between $I$ and $\tilde{B}$ on a subdomain $D \subseteq \mathcal{D}$ is measured by $\delta_{D}^{2}$, that is the sum of the $\Delta^{2}$ values for pixels belonging to the subdomain $D$. The value of $\delta_{D}^{2}$ is difficult to interpret in absolute terms. Thus, we consider the probability of observing $\delta_{D}^{2}$ by chance, denoted $\mathbb{P}_{H_{1}}\left(\delta_{D}^{2}\right)$. Hence, the NFA on a subdomain $D$ is defined by:

$$
\begin{aligned}
N F A_{1}(D) & =\eta_{1}(D) \mathbb{P}_{H_{1}}\left(\delta_{D}^{2}, \sigma\right), \\
& =\eta_{1}(D) \frac{1}{\Gamma\left(\frac{|D|}{2}\right)} \int_{0}^{\delta_{D}^{2} / 2 \sigma^{2}} e^{-t} t^{\frac{|D|}{2}-1} d t,
\end{aligned}
$$

where $\Gamma$ is the Euler function and $\eta_{1}(D)$ is a normalization term (equivalent to the number of tests) that controls the average number of false alarms. According to [8], it is sufficient that:

$$
\sum_{D \subseteq \mathcal{D}} \frac{1}{\eta_{1}(D)} \leq 1 .
$$

As in [18], we choose $\eta_{1}=|\mathcal{D}|\left(\begin{array}{l}|\mathcal{D}| \\ |D|\end{array}\right)$ to distribute the risk with respect to the size of the domain. Inequality 2 is satisfied since

$$
\begin{aligned}
\sum_{D \subseteq \mathcal{D}} \frac{1}{|\mathcal{D}|\left(\begin{array}{c}
|\mathcal{D}| \\
D \mid
\end{array}\right)} & =\frac{1}{|\mathcal{D}|} \sum_{n=|D| \leq|\mathcal{D}|}\left(\begin{array}{c}
|\mathcal{D}| \\
n
\end{array}\right) \times \frac{1}{\left(\begin{array}{c}
|\mathcal{D}| \\
n
\end{array}\right)}, \\
& =\frac{1}{\mathcal{D}} \sum_{n=|D| \leq|\mathcal{D}|} 1, \\
& =\frac{\mid \mathcal{D}}{\mathcal{D}}, \\
& =1 .
\end{aligned}
$$

Equation 1 dependencies to the parameters is thus

$$
N F A_{1}(D)=N F A_{1}\left(\delta_{D}^{2},|D|, \sigma,|\mathcal{D}|\right) .
$$

At the end of this first test of significance, we detected the subdomain $\hat{D}$ the most significant in term of $\Delta^{2}$ low pixel values. The parameters involved in Equation 1 are the cardinal of $|\mathcal{D}|$, that is directly accessible, and the standard deviation $\sigma$ of the naive model. This latter corresponds to the case of an image $\Delta$ totally unstructured, for example a difference between any pair of images (even not representing the same scene) or simply a difference between an image of the scene and a 'null' image (all pixels to 0 ). So $\sigma$ can be estimated from the standard deviation of $I$. 
By combining the results $\hat{D}$ with $\mathcal{D}$, we get a 3 -label image, where the subdomain of object pixels is $\mathcal{D} \backslash \hat{D}$, the subdomain of background pixels is $\hat{D}$ and the subdomain of unknown pixels $\overline{\mathcal{D}}$. In the following, a point is a pixel with label object and a point image is a label image where object pixels have been identified, as at the output of Algorithm 1 that describes the estimation of background pixel subdomain $\hat{D}$ by minimizing the $N F A_{1}$.

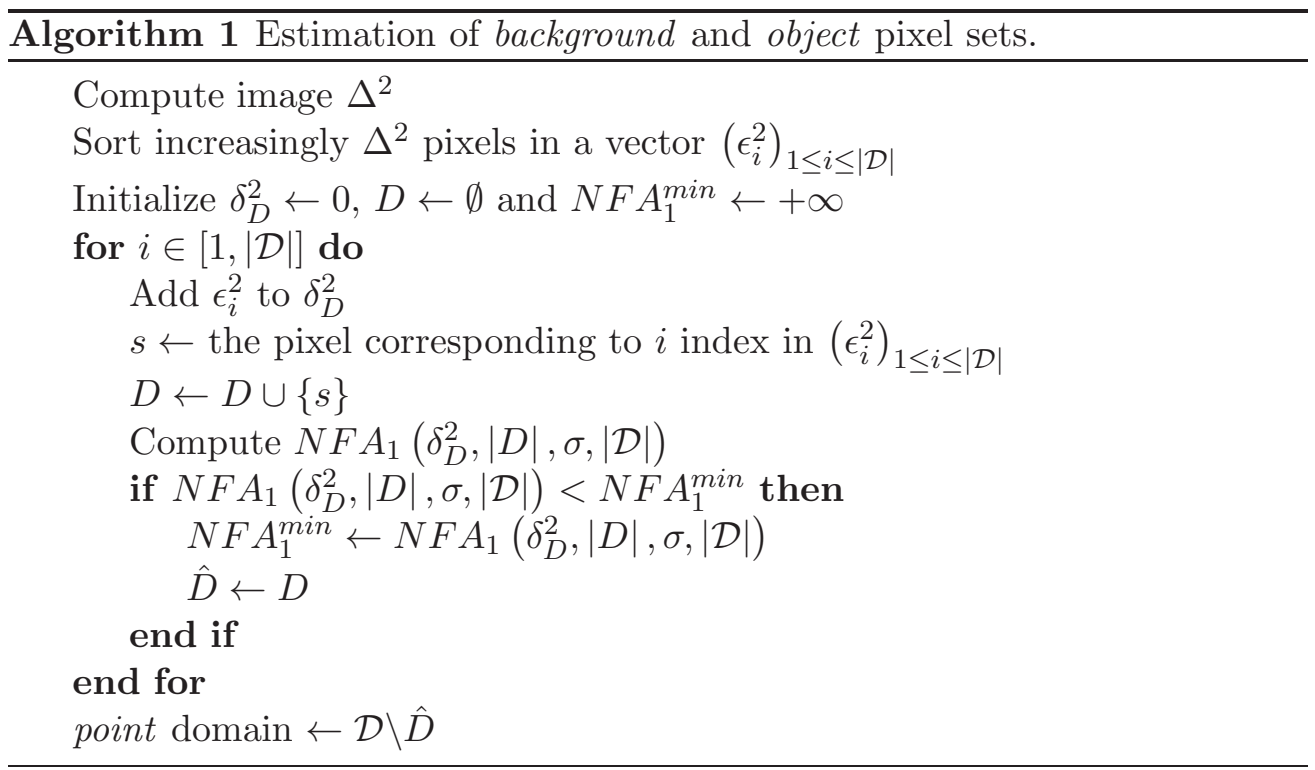

Figures 1a, 1b and 1c illustrate this algorithm in the case of a typical CCTV application. The aim was to detect the intrusions on the site and to classify the behaviors of the intruders in terms of dangerousness. The changes of interest are: (i) three people walking towards the camera, (ii) a fourth one running to the left, mid-hidden behind a car, (iii) a car on a road far away in the background of the scene. For this example, $\tilde{B}$ is simply an image acquired at the very beginning of the sequence. From Figure 1a, we note that these changes correspond to high pixel values in $|\Delta|$ image (on Figure 1a, the contrast has been enhanced for clarity). However, several other pixels exhibit such high values, probably because of variations in the background of the scene or in the cypress branches. Figure $1 \mathrm{~b}$ shows the curve giving the value of $\log \left(N F A_{1}\right)$ versus the cardinality of the domain $D$ (of pixels surprisingly consistent). It presents a clear minimum, while the curve of cumulative squared errors seems much more difficult to interpret automatically. Figure 1c shows the result of the detection at pixel level, namely $\mathcal{D} \backslash \hat{D}$ subdomain coded in white. Unknown 
pixels are absent in this example. We note that the four people and the car are quite well detected. But there is also a high number of isolated detections that illustrates the interest of taking into account a second criterion (on the density of the points).

Before introducing this second criterion, let us provide some comments about this first detection at pixel level. We can ask whether it would have been easier to directly detect the pixel significantly high values, defining differently the parameter $\sigma$ of the naive model, e.g. the standard deviation actually observed on $\Delta$. Two reasons argue against this model.

1. Considering $\delta_{D}^{2}$, i.e. a sum over $D$ pixels, makes more robust the detection. Now, assume that we search to detect the $D$ subdomain such that $\delta_{D}^{2}$ is particularly high. We will not be able to guarantee the minimum value of $\Delta^{2}(s), s \in D$ (since only one high value will make $\delta_{D}^{2}$ remains high, whatever the values added). Conversely, with our model, the maximum value of $\Delta^{2}(s), s \in D$ is bounded: $\forall s \in D, \Delta^{2}(s) \leq \delta_{D}^{2}$.

2. If there are unknown parameters, they can be estimated them according to a minimum error criterion. In [18], the parameters to be optimized were the class means. In the case of a moving camera (presented further), the camera roto-translation parameters will be optimized in the $6 \mathrm{D}$ motion space.

At the end of Section 2.2.3, we present an alternative to the presented detection at pixel level. Its interest is mainly theoretical: firstly because the induced computation time is a strong limitation, and secondly because of the interest of handling a double criterion, on pixel values (such as with $N F A_{1}$ criterion) and on point density (such as presented in next section).

\subsection{Level 2: Detection of Change Windows in the Image}

In this section, we process the point image, i.e. an image with only three labels: object, background and unknown, e.g. obtained at the output of the previous level. Under the assumption that the sought objects are big relatively to the pixels, we focus on the detection of clusters of points (pixels having the label object in the point image). In [6], two criteria were proposed.

Given a geometric shape (e.g. strip, window, or any'parametrized' compact domain), for each geometric object $O_{j}$ containing $n_{j}$ points, the first one is the NFA associated to the detection of geometric objects containing a number of points above or equal to $n_{j}$. The NFA criterion can be used either to detect all 


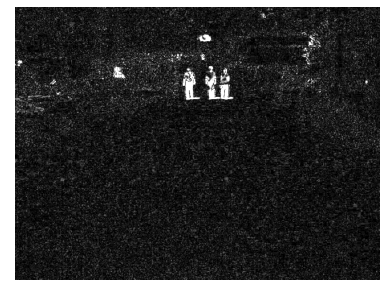

(a) $|\Delta|$ image

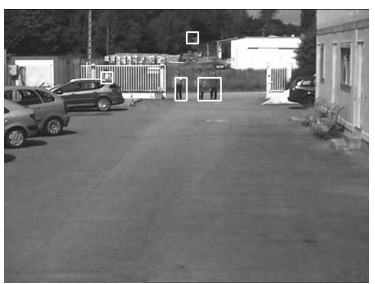

(d) Window level result

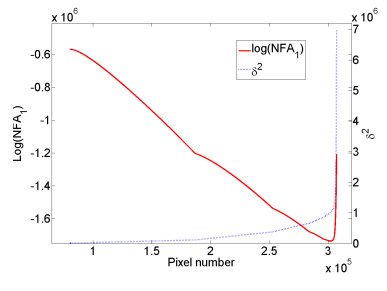

(b) $\log \left(N F A_{1}\right)$ and $\delta^{2}$ curves

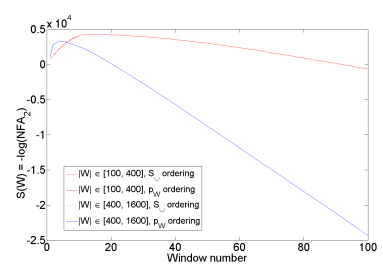

(e) $-\log \left(N F A_{2}\right)$ curve

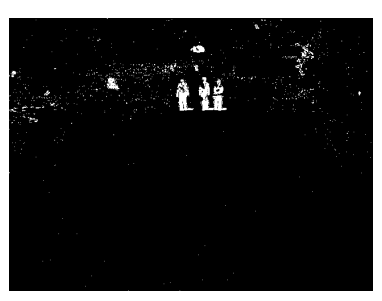

(c) Pixel level result

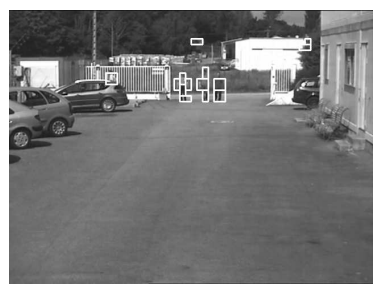

(f) Window level result

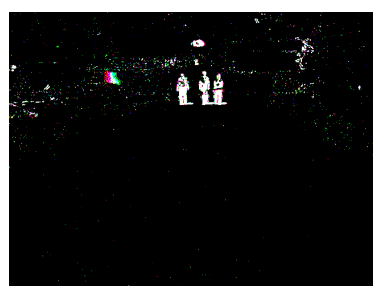

(g) Pixel level, 3 dates

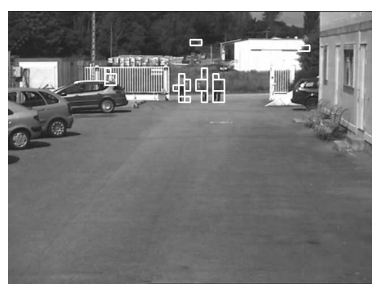

(h) Window level, 3 dates

Figure 1: Example of detection (static camera case): (a) Absolute difference image between foreground and background images, (b) curves of $\log \left(N F A_{1}\right)$ and $\delta^{2}$ (Algorithm 1) versus cardinality of $D$ (subdomain of amazingly consistent pixels), (c) result of detection at pixel level: 3label image (white $\equiv$ object, black $\equiv$ background), (d) result of detection at window level (medium window sizes), (e) curves of $-\log \left(N F A_{2}\right)(\mathrm{Al}-$ gorithm 2) versus cardinality of the window subset (amazingly dense windows), (f) result of detection at window level (small windows), (g) result of multitemporal detection at pixel level, (h) result of multitemporal detection at window level (small windows). 
the objects $O_{j}$ such that the associated NFA is below a given threshold, or to detect the most significant object(s).

The second NFA criterion [6] does not make any assumption on the geometric shape of the objects, except on the distance between the points within the object. Although it seems interesting to use clusters, two elements argue against in our application. First, for images with a large number of points, the computation time of the distance spanning tree is very long. Second, in the case of missing data (unknown label), the results are not stable. For instance, consider two clusters having distance parameters $r_{j}$ and $r_{j}-1$, separated by a distance $2 r_{j}-1$ and a pixel having unknown label located midway between clusters. Depending on this pixel label, the two clusters will merge into one or not. Thus, the results in terms of clusters detected can vary greatly depending on a single pixel label, that may be subject to errors.

In the following, we choose as a geometric shape, the rectangular window that allows approaching the shape of objects at low resolution. An interest of approach [9] is the ability to take into account a label unknown in the image. However, the proposed detection is not automatic since it uses a final threshold on NFA values (in addition to a first threshold for the creation of a 3-label image). The choice of the threshold being generally tricky and not robust, we propose to rather use a minimization of a NFA criterion. The naive model $\mathrm{H}_{2}$ is given by Definition 2 .

Definition 2 (naive model $H_{2}$ ). In the point image (labels $\in$ \{ object, background, unknown $\}$ ), the points (object labeled pixels) are uniformly independently distributed with probability $p$ and the background pixels are uniformly independently distributed with probability $1-p$.

According to Definition 2, for each window $W$ having $n$ defined pixels, the probability to contain more than $k$ points is given by the tail of the binomial distribution. Let $p$ be the probability of a point among defined pixels $\left(p=\frac{|\mathcal{D} \backslash \hat{D}|}{|\mathcal{D}|}\right.$ where $\mathcal{D}$ is the domain of defined pixels and $\mathcal{D} \backslash \hat{D}$ is the domain of points), $\kappa_{W}$ is the number of points in the considered window $W\left(\kappa_{W}=|W \cap \mathcal{D} \backslash \hat{D}|\right), \nu_{W}$ is the total number of defined pixels in $W\left(\nu_{W}=|W \cap \mathcal{D}|\right)$. Denoting $\mathbb{P}_{H_{2}}\left(\kappa_{W}\right)$ the probability of observing by chance a cluster of $\kappa_{W}$ points, a NFA measure on the window $W$ is given [9] by:

$$
\begin{aligned}
N F A_{2}(W) & =\eta_{2}(W) \mathbb{P}_{H_{2}}\left(p, \kappa_{W}, \nu_{W}\right), \\
& =\eta_{2}(W) \sum_{i=\kappa_{W}}^{\nu_{W}}\left(\begin{array}{c}
\nu_{W} \\
i
\end{array}\right) p^{i}(1-p)^{\nu_{W}-i},
\end{aligned}
$$

where $\eta_{2}$ is a number of tests. In [9], this number is a constant, thus the 
NFA measure is proportional to the probability. In [6], $\eta_{2}$ is the number of strips (or windows if we transpose for our case) necessary to cover the entire image, i.e. $\eta_{2}(W)=\frac{|\mathcal{D} \cup \overline{\mathcal{D}}|}{|W|}$ in our case. In order to penalize more severely the false detection, we propose $\eta_{2}(W)=\frac{|\mathcal{D} \cup \overline{\mathcal{D}}|}{|W|} \times 2^{|W|}$, which also satisfies the condition $\sum_{j} \frac{1}{\eta_{2}\left(W_{j}\right)} \leq 1$.

Defining the point probability within $W$ by $p_{W}=\frac{\kappa_{W}}{\nu_{W}}$, the significance $S\left(p, p_{W}, \nu_{W},|W|\right)=-\log \left(N F A_{2}(W)\right)$, can be written using the Hoeffding approximation:

$$
\begin{aligned}
S\left(p, p_{W}, \nu_{W},|W|\right) \approx & \nu_{W}\left[p_{W} \ln \left(\frac{p_{W}}{p}\right)+\left(1-p_{W}\right) \ln \left(\frac{1-p_{W}}{1-p}\right)\right] \\
& -\log \left(\eta_{2}(W)\right) .
\end{aligned}
$$

Following [9], when windows are partially overlapping, only the one having the maximum significance should be kept. For this, we sort the windows $W_{j}$ by decreasing significance, and we scan the list of sorted $W_{j}$, removing any window $W_{k}$ overlapping the current $W_{j}$ and further in the list (i.e. having lower significance). Therefore, at the end of this step, only non overlapping windows having 'maximal signficance' remain in the sorted list.

\subsubsection{Change window Subset}

In [9], the selected windows are those presenting a significance value greater than a threshold value $S_{\text {min }}$. However, the choice of this minimal significance is hardly less tricky than a threshold on probability (actually for a constant test number $\eta_{2}$ it is equivalent). Now one strong advantage of the measure in term of NFA is to be a number, that is to say directly comparable to other NFA values. Thus, we propose to compute the NFA of window subsets in order to minimize the it, or equivalently to maximize the significance over the window subsets.

Consider the case where all the windows have same number of defined pixels and there is no overlapping between the windows. We search the subset of windows, denoted $\left\{W_{j}\right\}_{j=1}^{k}$, that maximizes the significance (under the naive model $\mathrm{H}_{2}$ ). The window indices correspond to the order in which the windows have been added to the current window subset: for a given window subset, $j \in$ $\{1, \ldots, k-1\}$, the $k$ index window is the window that maximizes the significance among all subsets of $k$ windows: $W_{k}=\operatorname{argmax}_{W}\left\{S\left(p,\left\{W_{1}, \ldots, W_{k-1}, W\right\}\right)\right\}$. For windows of same number of defined pixels, the $k$ windows maximizing 
the significance $S\left(p,\left\{W_{j}\right\}_{j=1}^{k}\right)$ at given $k$ value, are the $k$ windows having the greatest $p_{W}$ values: $\left\{W_{j}\right\}_{j=1}^{k}$ such that $p_{W_{1}} \geq p_{W_{2}} \geq \ldots \geq p_{W_{k}}$. The demonstration is trivial from Equation 6 presented just after. Let us note $\nu_{1} \ldots k$ the number of defined pixels within the window subset $\left\{W_{j}\right\}_{j=1}^{k}$ and $\overline{p_{k}}$ the probability for a pixel to be a point locally to $\left\{W_{j}\right\}_{j=1}^{k}: \overline{p_{k}}=\frac{\kappa_{1 \ldots k}}{\nu_{1 \ldots k}}$, with $\kappa_{1 \ldots k}$ the number of points within $\left\{W_{j}\right\}_{j=1}^{k}$. Equation 5 becomes:

$$
\begin{aligned}
S\left(p,\left\{W_{j}\right\}_{j=1}^{k}\right)= & -\log \left(N F A_{2}\left(\left\{W_{j}\right\}_{j=1}^{k}\right)\right) \\
\approx & \nu_{1 \ldots k}\left[\overline{p_{k}} \log \left(\frac{\overline{p_{k}}}{p}\right)+\left(1-\overline{p_{k}}\right) \log \left(\frac{1-\overline{p_{k}}}{1-p}\right)\right] \\
& -\log \left(\eta_{2}\left(\left\{W_{j}\right\}_{j=1}^{k}\right)\right),
\end{aligned}
$$

where $\eta_{2}\left(\left\{W_{j}\right\}_{j=1}^{k}\right)$ is the number of tests associated to the considered window subset. We choose $\eta_{2}\left(\left\{W_{j}\right\}_{j=1}^{k}\right)=\frac{|\mathcal{D}|}{\nu_{1 \ldots k}} 2^{\nu_{1 \ldots k}}$ to take into account the fact that within a window subset of cardinality $\nu_{1 \ldots k}$, there are $2^{\nu_{1 \ldots k}}$ possible subsets of points. This term satisfies Inequality 2 provided that the windows are large enough. Precisely, denoting $a$ a lower bound for the average number of pixels within a window $W_{j}$ and $N_{W}$ is the total number of possible windows:

$$
\begin{aligned}
\frac{1}{|\mathcal{D}|} \sum_{k=1}^{N_{W}} \frac{\nu_{1} \ldots k}{2^{\nu_{1} \ldots k}}\left(\begin{array}{c}
N_{W} \\
k
\end{array}\right) & \leq \frac{1}{|\mathcal{D}|} \sum_{k=1}^{N_{W}} \frac{a k}{2^{a k}}\left(\begin{array}{c}
N_{W} \\
k
\end{array}\right), \\
& \leq \frac{a}{|\mathcal{D}|}{\frac{N}{2_{W}}}^{a}\left(1+\frac{1}{2^{a}}\right)^{N_{W}-1}, \\
& \leq \frac{a}{|\mathcal{D}|} \frac{N_{W}}{2^{a}} e^{\frac{N_{W}-1}{2^{a}}} .
\end{aligned}
$$

Previous inequality was obtained using successively the equality $\forall x \in \mathbb{R}$, $\forall n \in \mathbb{N}, x \frac{d}{d x}(1+x)^{n}=\sum_{k=1}^{n} k x^{k}\left(\begin{array}{l}n \\ k\end{array}\right)$ and the inequality $\forall x, r \in \mathbb{R}^{+},(1+x)^{r} \leq$ $e^{r x}$. The solving of the last right member of the inequality can be done using the Lambert $\mathrm{W}$-function (main branch). Anyway, in our case, the inequality is widely checked since $|\mathcal{D}|=640 \times 480, a \approx 10 \times 10$ and $N_{W} \approx 64 \times 48 \times 4 \times 4$.

In the case of windows with different numbers of defined pixels, the two orderings in probability and significance may differ, so that we theorically should test any window combination. Specifically, for every number of defined pixels, we have to create a list of windows with the same number of defined pixels and sorted by $p_{W}$. Then, for every list, we compute the significance of the current window subset added with the top window in the list, and we choose the window maximizing the significance (and move it to the window subset). Since it may be intractable and since our tests (see Figure 1e) show the difference is 
very small, we propose a simplified version using the $p_{W}$ ordering to construct $\left\{W_{j}\right\}_{j=1}^{k}$.

Algorithm 2 describes the estimation of the subset of windows by maximizing the significance. The first part of the algorithm eliminates the non-maximal significance windows. The second part of the algorithm aims at detecting the subset of non-overlapping windows having maximal significance. As said previously, we limit the number of tested window subsets to the number of remaining windows at the end of the first step (adding, one by one, the windows to the current subset). Such a process is optimal if and only if the number of defined pixels by window is the same. In our case, it is thus suboptimal. Note also that the algorithm does not use any threshold parameter.

Figure 1d presents results on test images previously considered in the case of variable 'medium' size windows (width $\times$ height) $20 \times 20,20 \times 40,40 \times 20$ and $40 \times 40$. Note that the choice of the maximum or minimum size of a window depends on an implicit assumption on the minimum size of the researched objects. Thus, both for computation time reasons and for a priori about the minimum size of sought objects, we do not explore all window sizes. From Figure 1d, the results obtained are satisfying since the four people and the car are detected. Figure $1 \mathrm{f}$ shows results in the case of variable 'small' size windows $10 \times 10,10 \times 20,20 \times 10$ and $20 \times 20$. We note that objects of interest are now discerned in a finer way at the price of two false detections in the cypress.

Figure 1e compares the two ways to construct the window subset $\left\{W_{j}\right\}_{j=1}^{k}$ : the optimal one (i.e. sorting by significance) or the simplified one (i.e. sorting by $p_{W}$, cf. Algorithm 2). It shows the four significance curves corresponding to each construction way and to each set of window size, 'small' and 'medium'. From Figure 1e, the difference between the optimal subset estimation and the simplified one is unnoticeable (in fact it only occurs at the very beginning of the curves). Note that for both window subset construction, the NFA curves show a clear extremum (NFA minimum or significance maximum) allowing a parameter-free algorithm. We insist on the fact that, when using Algorithm 2 the number of detected windows is derived from a maximization and not from a threshold criterion. Now, there is maximum in any case, regardless of the presence of an actual object. So, rigorously, there is an implicit threshold parameter on the maximum significance value obtained: in order to ensure less than 1 false alarm (in expectation), the maximum significance value should be greater than 0 . 


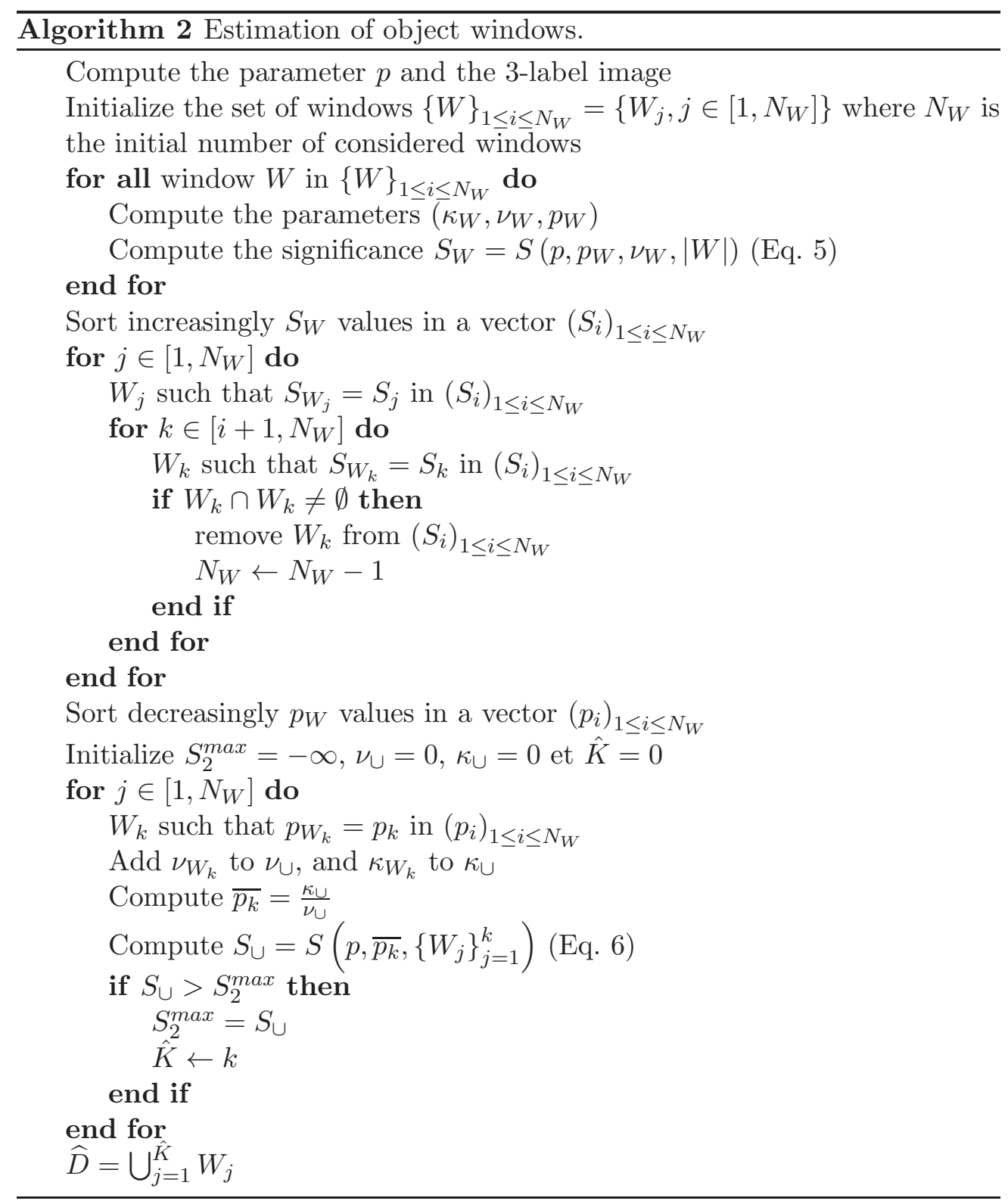

\subsubsection{Multitemporal Case}

We propose an extension of previous measure to the case of multidimensional point images. The typical applicative case is the multitemporal one: not only 
one pair of images acquired at $(t-1, t)$ is considered but a set of image pairs, e.g. acquired at $(t-3, t-2),(t-2, t-1),(t-1, t)$. The extension of $N F A_{2}$ criterion is as follows. Let be $N$ point images, the algorithm parameters are computed on 3D domains, such that the two first dimensions are those of the previously considered 2D domains and the third dimension is the dimension of $N$, i.e. the temporal dimension in our case. Algorithm 2 is still valid except that the handled windows are now $3 \mathrm{D}$ windows (in particular their cardinality in $2 \mathrm{D}$ is multiplied by $N)$ and the parameters $\left(p, \kappa_{W}, \nu_{W}\right)$ are now defined by:

$$
\begin{aligned}
p & =\frac{\sum_{t=1}^{N}\left|\mathcal{D}_{t} \backslash \hat{D}_{t}\right|}{\sum_{t=1}^{N}\left|\mathcal{D}_{t}\right|}, \\
\kappa_{W_{j}} & =\sum_{t=1}^{N}\left|W_{j} \cap \mathcal{D}_{t} \backslash \hat{D}_{t}\right|, \\
\nu_{W_{j}} & =\sum_{t=1}^{N}\left|W_{j} \cap \mathcal{D}_{t},\right|,
\end{aligned}
$$

where the index $t$ refers to the point image among the $N$ point images. Adding a third dimension has the effect of strengthening the 2D detections correlated along this third dimension. Thus, the temporal dimension appears as a good candidate for object detection, conversely to the color for instance. However, note that in the case of a moving object, as in the case of a moving camera, optimal results will be obtained only by considering the object motion and/or by compensating the camera egomotion.

Figure $1 \mathrm{~g}$ shows the result of multitemporal detection at pixel level for 3 point images, each one being coded with a primary color (red, green and blue), so that the composite colors (yellow, magenta, cyan and white) denote multiple detections. Figure $1 \mathrm{~h}$ shows the result of multitemporal detection at window level, i.e. using Algorithm 2 with modified Equations 8. For comparison the monotemporal result was shown on Figure 1f. Among the two false alarms observed in the monotemporal case, one has disappeared in the multitemporal case. We also note that two people are better discerned, their head being now detected. Note that in the case of the 'medium' size windows (Figure 1d), the results obtained for $N=1$ and $N=3$ are the same, because spatial 'filtering' is already adequate.

\subsection{3. $N F A_{1}$ Criterion Against $N F A_{2}$ Criterion}

Algorithm 1 (Section 2.1) is similar to a step at pixel level which produces a point image. A fundamental property of NFA measurements is to be com- 
parable. This allows an alternative approach using $N F A_{2}$ criterion to detect directly from the grayscale images, as follows.

Let simply threshold the images $|\Delta|$ varying the threshold parameter, called $\tau$, and obtain as many point images resulting of this $|\Delta|$ image thresholding. For each point image associated to a $\tau$ value, calculate the $N F A_{2}$ assuming the binomial distribution for naive model. The optimal threshold for a window $W$ is then the $\tau$ value maximizing $S\left(p(\tau), p_{W}(\tau), \nu_{W}(\tau),|W|\right)$ where the dependencies of parameters to $\tau$ are shown. Then, $\tau_{W}=\operatorname{argmax}_{\tau} S\left(p(\tau), p_{W}(\tau), \nu_{W}(\tau),|W|\right)$.

Even if such an approach seems unrealistic with the actual embedded computers, we illustrate it on one result because of its theoretical interest. Figure 2a shows, for six specific windows, the curves of the $S(W)$ versus the threshold $\tau$. We clearly see a maximum that is all the more significant that the window effectively contains an object (or an object subpart). In the expression 'Window $(a, b)$ ', $a$ denotes the window upperleft corner row index and $b$ the window upperleft corner column index. Thus, the subplots 'Window $(120,200)$ ', 'Window(120,280)' and 'Window(120,320)' correspond to three people and the 'Window $(160,0)$ ' to a parked car. 'Window $(120,0)$ ' and 'Window $(40,480)$ ' are false alarms. Figure $2 \mathrm{c}$ shows the result of the detection when applying the detection of the optimal threshold according to $N F A_{2}$ criterion to windows realizing a partition of the image. The result seems promising.

However, we waived this approach for two reasons. First, even it seems unsupervised (since the 'optimal' threshold parameter is automatically estimated), it contains a supervised parameter: the threshold to decide if the maximum of significance is significant. Second, using only $N F A_{2}$ criterion bases the detection on the sole criterion of amazingly high relative density of points, whereas using $N F A_{1}$ criterion (as a first step) introduces a criterion based on observed radiometric values. In the following of the study, we base detection on a double criterion, namely criteria $N F A_{1}$ and $N F A_{2}$.

\section{Variants of Proposed Approach}

In the previous section, the methodology was developed and tested in the case of a static camera. Here, we extent it to the case of a camera in motion that is a typical case of driver assistance system applications. The aim is to illustrate the genericity of the presented approach with respect to the input data. 

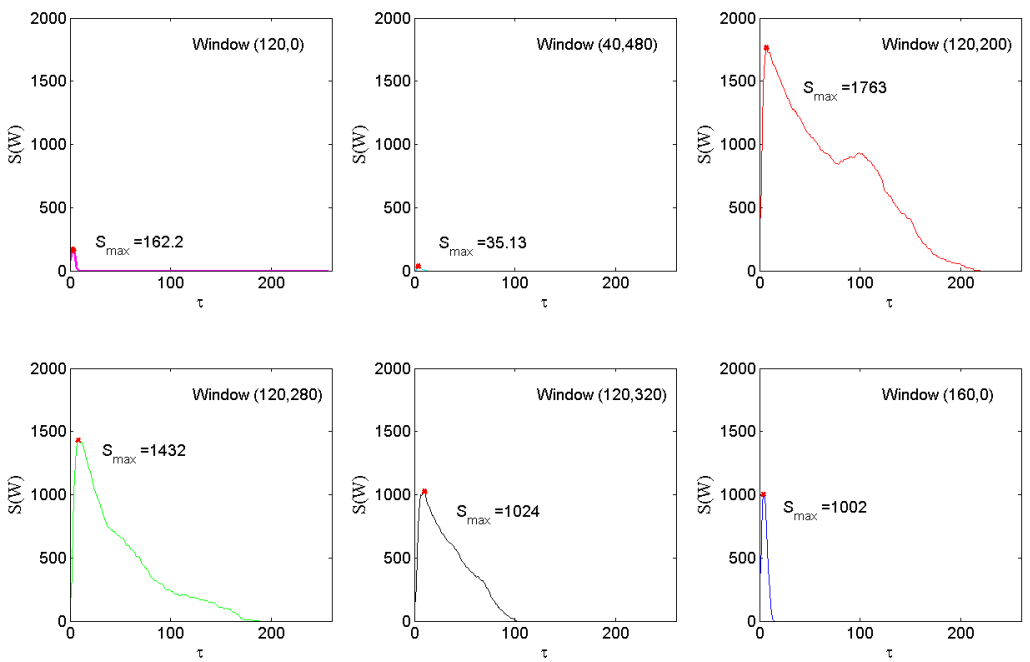

(a) $-\log \left(N F A_{2}\right)$ curves, 6 window cases

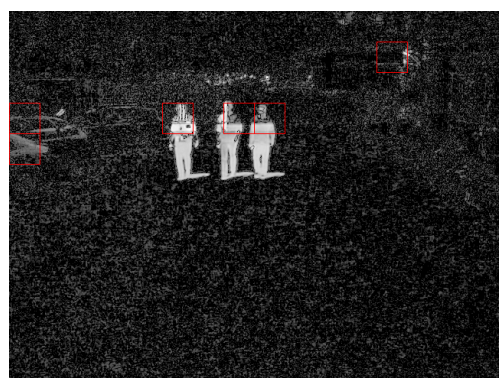

(b) 6 window cases

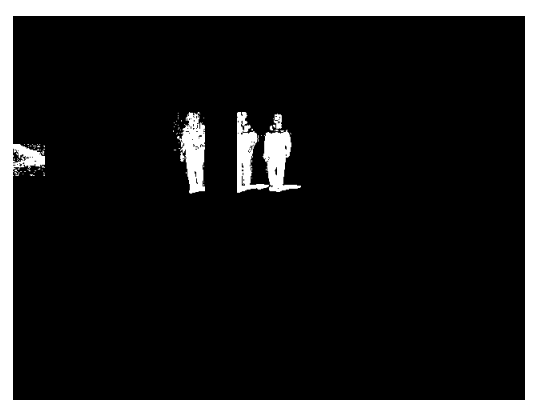

(c) Mosaic of $N F A_{2}$ criterion thresholded windows

Figure 2: Example of pixel detection according to $N F A_{2}$ criterion (gray-level image case): (a) significance curves versus threshold parameter $\tau$ for six cases of windows, (b) $|\Delta|$ image indicating the six considered windows, (c) mosaic of the thresholded windows using threshold parameter maximizing the significance, provided that significance maximum is greater than 500 . 


\begin{tabular}{|c|c|c|c|}
\hline & & \multicolumn{2}{|c|}{ innovation } \\
\hline & & radiometric image & disparity image \\
\hline 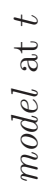 & $\begin{array}{l}\text { proj. radiometric im. } \\
\text { proj. disparity im. } \\
\text { blank scene disparity }\end{array}$ & $\begin{array}{c}\text { moving objects } \\
/ /\end{array}$ & $\begin{array}{c}\text { / } \\
\text { moving objects } \\
\text { salient objects }\end{array}$ \\
\hline
\end{tabular}

Table 1: Kind of detected objects versus input data.

\subsection{Three Declinations of the Proposed Approach}

Our objective is the detection of objects in a video sequence using a camera embedded on a vehicle, generally moving. In the outdoor scene, the objects of interest are usually pedestrians, other vehicles and obstacles on the road or nearby (walking on the sidewalk and likely to cross the road). Since the a-contrario approach does not base on the characteristics of the sought objects, but on the noise model, only the input data may include the a priori information on the objects of interest. In this study, we propose to detect different kinds of objects simply using different input data to derive the image $\Delta$ at the input of the proposed algorithm.

\subsubsection{Detected Objects Versus Input Data}

For the detection of moving objects, the input images are the image at time $t$ and the projection of the previous image in the geometry of the current one, in order to compensate for the egomotion of the camera and focus on moving objects. Images can either be usual images, here called radiometric images or disparity images. In the following, the term 'grayscale' image refers to a generic image with more than two or three values (i.e. not a binary or ternary image), without prejudging the physical meaning of these values (e.g. radiometry or disparity).

For the detection of static objects, the image $\Delta$ is derived from the image at the instant $t$ and a model of a 'blank' scene, i.e. without any object but the road. Due to the construction of the blank scene, only the case of disparity images is considered in this study. Table 1 summarizes the characteristics of the detected objects according to the combinations of input data, with in column the model at $t$ and in row the innovation at $t$. The following paragraphs specify these combinations. 
First type of model $t$ : a priori 3D scene In this first model, the background image is a blank scene but the road. The road is modeled as a part of a plane estimated directly from the analysis of the v-disparity [13]. In the a priori scene, each $3 \mathrm{D}$ point satisfies the equation of the road, or is located at infinity. Therefore, the model at $t$ we are able to construct is necessarily a disparity image (and not a radiometric image), noted $d_{\text {road }}$ in the following.

Noting $d_{t}$ the disparity image at $t$ that is the innovation here, $\Delta$ image is such that: $\Delta(s)=d_{t}(s)-d_{\text {roadt }}(s)$, with $s$ a pixel, $d_{t}(s)$ the value of the disparity for $s$ and $d_{\text {roadt }}(s)$ the value of the disparity for $s$ in the assumed blank scene except the road.

Second type of model $t$ : projection of the image at $t-1$ In this second model, the background image is deduced from the observed scene at the previous instant, namely $t-1$. As the camera is moving, the observed image at $t-1$ should be corrected for this camera motion. Assuming the camera and the vehicle are rigidly linked, we build the background from the gray-level image at $t-1$, the disparity map at $t-1$, the 6 motion parameters of the camera, namely the translation $T=\left(T_{X}, T_{Y}, T_{Z}\right)$ and the rotation $\Omega=\left(\Omega_{X}, \Omega_{Y}, \Omega_{Z}\right)$, supposed constant between $t-1$ and $t$. The smaller the motion (in particular the rotational component), the more accurate is the projection of the image. The output of the projection of $(t-1) \rightarrow t$ is then the model at $t$. Now, these images contain missing values in some pixels, firstly due to missing values in the disparity map at $t-1$ and secondly due to the change in the field of view (due to camera motion).

First kind of innovation at $t$ : radiometric images. Considering radiometric images, $\Delta$ image is defined by: $\Delta(s)=I_{t}(s)-I_{t-1 \rightarrow t}(s)$ with $s$ a pixel, $I_{t}(s)$ the radiometric value in $s$ at $t$, and $I_{t-1 \rightarrow t}(s)$ the radiometric value in $s$ after projection of the image $I_{t-1}$ acquired at $t-1$ (knowing the $6 \mathrm{D}$ motion of the camera).

Second kind of innovation at $t$ : disparity images. The $\Delta$ image is here defined by: $\Delta(s)=d_{t}(s)-d_{t-1 \rightarrow t}(s)$ with $s$ a pixel and $d_{t-1 \rightarrow t}(s)$ the disparity value in $s$ after projection of the disparity map $d_{t-1}$ acquired at $t-1$ (knowing the $6 \mathrm{D}$ motion of the camera).

\subsubsection{Example Comparing the Three Declinations}

In order to compare our three declinations, we use a specific ADAS dedicated software, Pro-SiVIC, that can model and render quite complex road scenes using sophisticated camera models and can provide the ground true about odometric data, actors positions and deep/disparity data. A typical scenario includes 
some buildings along the road, a near approaching car and two pedestrians at the crosswalk. There is also a car very far in the background of the scene and another pedestrian also far away. The stereo camera pair is embedded on a vehicle driving at about $36 \mathrm{~km} / \mathrm{h}$ and produces the primary video flow used in our simulation. Figure 3 presents the results obtained respectively for the three combinations of data input and model at $t$. We observe that:

- Radiometric data lead to point images, at the output of Algorithm 1, with more false detections (at pixel level) than the disparity data that tend rather to sub-detect. After detection at window level using Algorithm 2, the results are very similar with a slight underdetection in the case of the disparity data. In both cases, only the oncoming car is detected, but neither the pedestrians, probably because they are too slow, and therefore seen as static objects, nor the car in the scene background, probably because it is too small in term of pixel number and its apparent motion in the image is too small.

- Using a blank scene (but the road) model allows the detection of vertical objects, namely buildings, the car in front and the two pedestrians. The car in the scene background and the pedestrian far away are not seen, probably because of their proximity (in term of distance in pixels) from the plane of the road.

First note that using radiometric images leads to results both less robust and more accurate than the use of images of disparity (in the case where the model at $t$ is the image at $t-1$ projected at $t$ ). Let us further comment on this observation.

Concerning the disparity images, three points have to be specified. First, the lack of precision on the disparity maps is due to block matching process between each image of the stereo pair, based on a SAD (Sum of Absolute Difference) minimization. Thus it induces a loss of spatial resolution of the order of block size used.

Second, the disparity $d_{m}$ varies with $\frac{1}{Z_{M}}$ where $Z_{M}$ is the depth coordinate: $d_{m}=\frac{f b_{s}}{Z_{M}}$ with $f$ the camera focal and $b_{s}$ the baseline between the cameras. Thus, the closer an object moving along the depth axis, the more important is the change in disparity values. For instance, for a car at $10 \mathrm{~m}$ in front of the camera moving away at a speed until $50 \mathrm{~km} / \mathrm{h}, b_{s}=0.5 \mathrm{~m}, f=780 \mathrm{pix}$., as in our simulated data, and a video recording $30 \mathrm{im} . / \mathrm{s}$, the variation of the disparity values of the pixels inside the car is less than 2 pixels. Now for the whole scene, the disparity values vary from 0 to about 50 according to the 


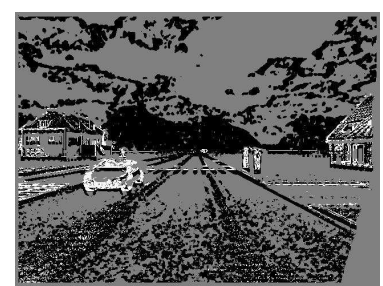

(a)

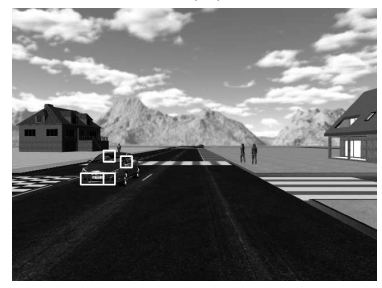

(d)

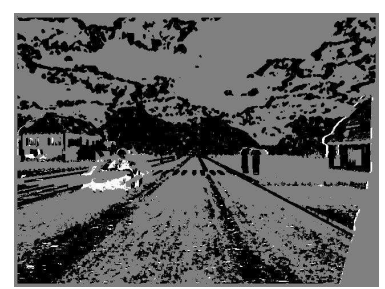

(b)

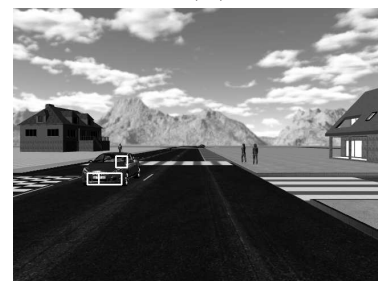

(e)

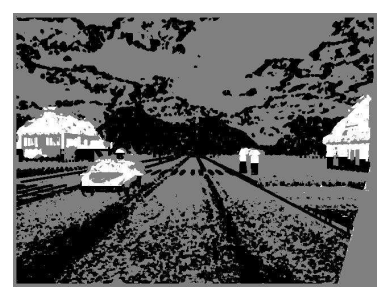

(c)

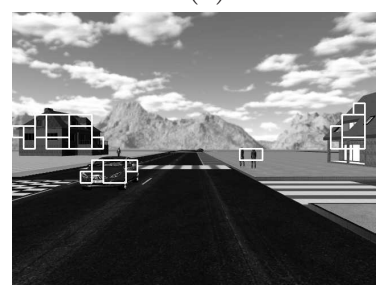

(f)

Figure 3: Result example with simulated data: $1^{\text {st }}$ line: pixel detection at the output of Algorithm 1 with 3 labels, namely white $\equiv$ object, black $\equiv$ background and gray $\equiv$ unknown (pixels where the disparity value is unknown); $2^{\text {nd }}$ line: window detection at the output of Algorithm 2; $1^{\text {st }}$ column: input data $\equiv$ radiometric images, model at $t \equiv$ projection of the image at $t-1 ; 2^{\text {nd }}$ column: input data $\equiv$ disparity images, model at $t \equiv$ projection of the image at $t-1 ; 3^{\text {rd }}$ column: input data $\equiv$ disparity images, model at $t \equiv$ blank scene but the road.

distance $Z_{M}$ relative to the camera. Moreover, the disparity is mainly available on the object vertical edges. Thus, the detected changes in the disparity values mainly correspond to occultation/uncovering of the background of the scene (or of objects in the background), i.e. they mainly occur at the borders of the objects.

Third, in the images of the sequence Pro-SiVIC, the disparity maps are rather 'clean', i.e. they contain only few errors: in particular the disparity values in the sky are absent rather than wrong (values in gray on the Figure 3, $1^{\text {st }}$ line). In less favorable (and more realistic) cases, there may be errors (e.g. in the sky) causing false detections on the point image. Multitemporal approach is then interesting to remove these false detections (provided that they are temporally decorrelated).

In conclusion, the use of the disparity coupled with a model at $t$ which is the previous image (projected) is robust and more prone to non detections 
(because of the smallness of disparity variations out of occultation/uncovering phenomena) provided that the disparity maps have sufficient quality (even be less dense). In the case of a noise on the disparity values, adding a multitemporal dimension acts as a multitemporal filter and significantly reduces the number of false detections as long as they are temporally uncorrelated. However, in the case where the object is moving so that there is no overlapping between its positions at successive instants, the filter leads to false negatives (non detections).

\subsection{Accurate Estimation of the Ego-Motion}

In our case application, the camera is in motion and the $6 \mathrm{D}$ motion of the camera is a parameter of the image model. However, there may be several causes of inaccuracy in the measurement of this movement: the intrinsic precision of odometers, the data synchronization (between camera and odometers: even with a same acquisition frequency for the different sensors, there may be a difference in the times of acquisition of the order of several tens of milliseconds). Now, the projection of the image at time $t-1$ to time $t$ is very sensitive to these parameters. Here, we propose to use the criterion $N F A_{1}$ to estimate simultaneously the points and the precise parameters of the motion. We use an initialization of researched parameters because seeking the motion in the whole $6 \mathrm{D}$ space would be intractable. Algorithm 3 describes the optimization of these parameters simultaneously with the estimation of points sub-domain by minimizing the $N F A_{1}$.

To illustrate the joint estimation of points and precise movement of the camera, Figure 4 shows an example with Pro-SiVIC simulated radiometric images: at time $t-1$ (Figure $4 \mathrm{a}$ ) and at time $t$ (Figure $4 \mathrm{~d}$ ). We note the movement of the camera visible on the positions of the road markings in the two respective images. Figure $4 \mathrm{~b}$ and $4 \mathrm{c}$ show the image 'projected' to $t$ either using the rough values (noted $\left(T_{0}, \Omega_{0}\right)$ ) of the $6 \mathrm{D}$ motion or the optimized values $(\hat{T}, \hat{\Omega})$. In both cases, we note the presence of undefined values due to some defaults in the disparity maps. Even if difficult to perceive visually, the differences between these two projections are present as shown by the subsequent results. Figure 4e and $4 \mathrm{f}$ show the results of the point detection using $\left(T_{0}, \Omega_{0}\right)$ and $(\hat{T}, \hat{\Omega})$, respectively. The number of false alarms present in the absence of optimization decreases significantly thanks to parameter optimization. Figure $4 \mathrm{~g}$ shows the $N F A_{1}$ curves versus the cardinal of the domain $D$ (pixels surprisingly consistent) respectively with and without optimization of $6 \mathrm{D}$ motion. We check that 


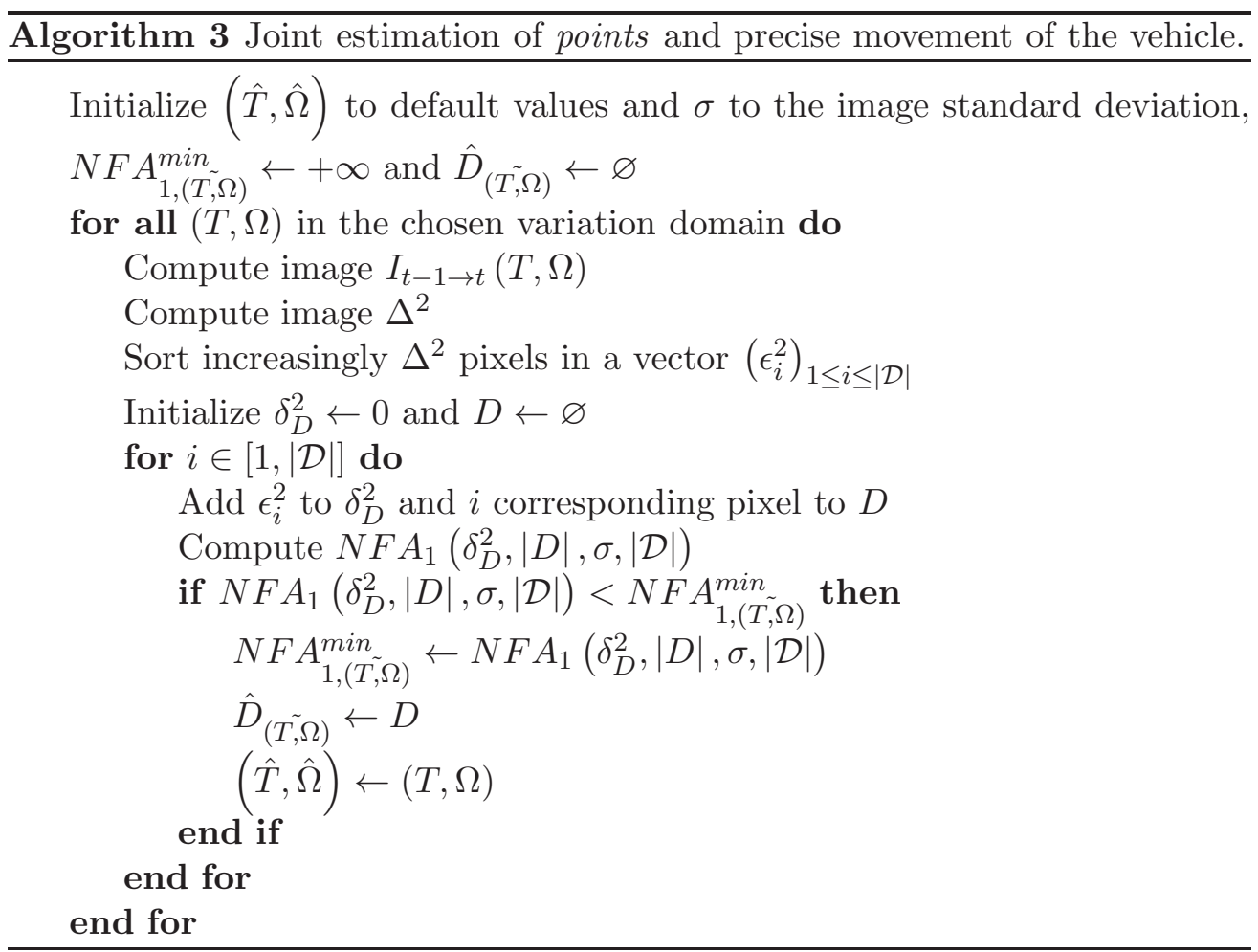

the curve with optimization is below. Finally, Figures $4 \mathrm{~h}$ and $4 \mathrm{i}$ show the result of the detection at window level obtained using $\left(T_{0}, \Omega_{0}\right)$ and $(\hat{T}, \hat{\Omega})$, respectively. Relatively to the case of the use of the rough $6 \mathrm{D}$ motion parameters, two false alarms have been removed, namely at the borders of the road marking (some others remain at the pedestrian crossing level) and on the house window. The cars are also slightly better detected, even if the car in front of the camera remains only poorly detected (due to its movement very close to the camera one).

We also tested the movement optimization in the case of disparity images. We found that, in most of the cases, the optimization do not improve the results, probably due the intrinsic imprecision of the disparity images, which can thus be interpreted here as a factor of robustness. 


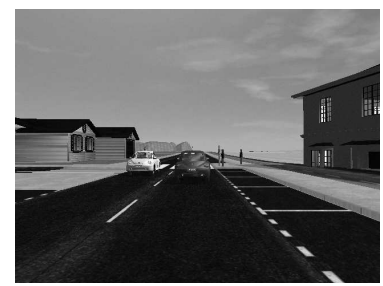

(a) Image at $t-1$

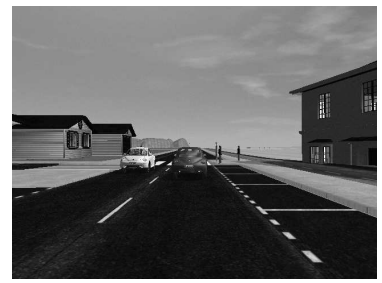

(d) Innovation at $t$

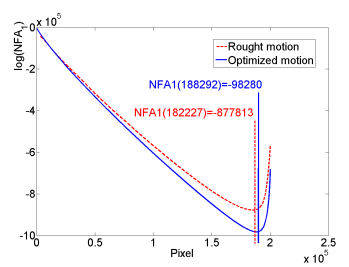

(g) $\log \left(N F A_{1}\right)$ curves

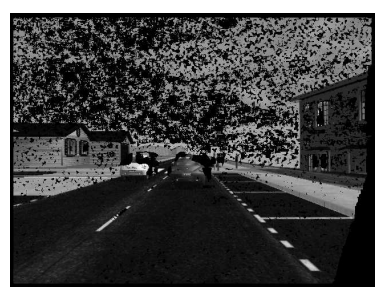

(b) Rough model at $t$

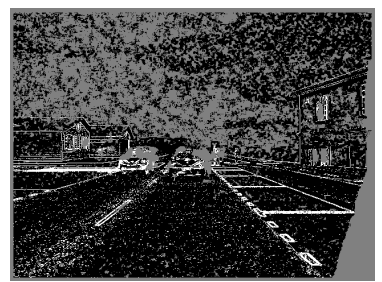

(e) point im./(T0, $\left.\Omega_{0}\right)$

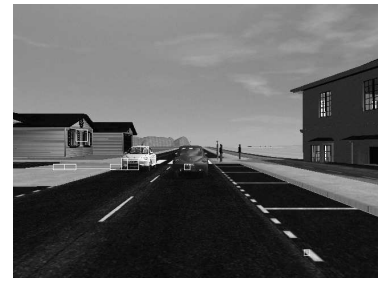

(h) wind. change/ $\left(T_{0}, \Omega_{0}\right)$

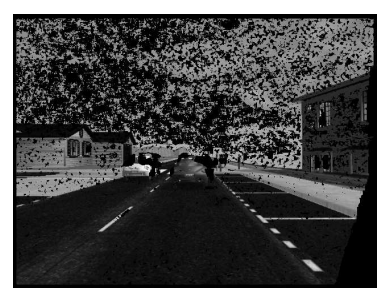

(c) Optimized model at $t$

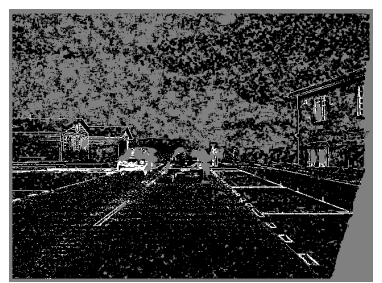

(f) point im./( $\hat{T}, \hat{\Omega})$

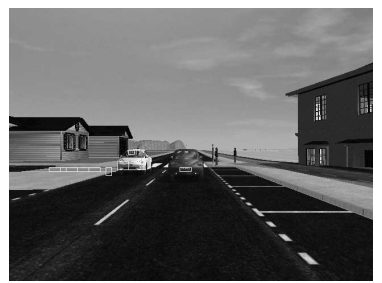

(i) wind. change $/(\hat{T}, \hat{\Omega})$

Figure 4: Example with 6D motion optimization: (a) image at $t-1$, (b-c) projections (undefined pixels are set to 0 ) of the image at $t-1$ that plays the role of the model at $t$ obtained (b) using rough $\left(T_{0}, \Omega_{0}\right)$ values, (c) using optimized $(\hat{T}, \hat{\Omega}),(\mathrm{d})$ image at $t$ that plays the role of

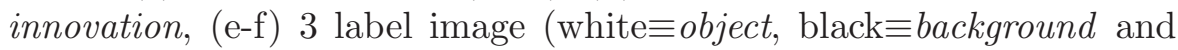
gray $\equiv$ unknown) obtained (e) using rough $\left(T_{0}, \Omega_{0}\right)$ values, (f) using optimized $(\hat{T}, \hat{\Omega}),(\mathrm{g})$ curves of $\log \left(N F A_{1}\right)$ versus $|D|$, (h-i) result of detection at window level using $(\mathrm{h})$ rough $\left(T_{0}, \Omega_{0}\right)$ values or (i) using $(\hat{T}, \hat{\Omega})$. 


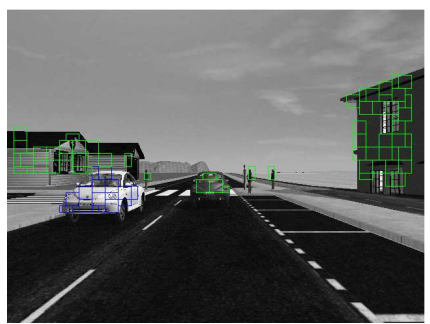

(a) radiom./disp. result

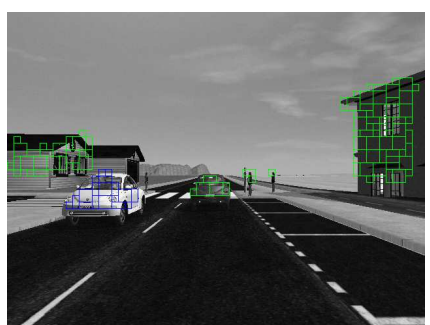

(b) disp./disp. result

Figure 5: Classification example with simulated data: 2 labels, namely green $\equiv$ static object, blue $\equiv$ moving object; $\left\{O_{V e}\right\}$ estimated from disparity images versus blank scene but road, $\left\{O_{C h}\right\}$ estimated from (a) radiometric images, (b) disparity images.

\subsection{Classification Based on Result Crossing}

In Section 3.1, we compare the results obtained from different declinations of our approach. We saw that using as model at $t$ a blank scene except the road and using as model at $t$ the projection of the image from $t-1$ instant to $t$ lead to complementary results. We propose to combine these two declinations to distinguish between static objects, i.e. in $\left\{O_{S t}\right\}$, and moving objects, i.e. in $\left\{O_{M o}\right\}$. Very simply, the model using a blank scene except the road provides the objects out of the plane of the road, either static or moving, while the model using the projection of the $t-1$ image provides only objects with own motion (and some false detections), i.e. in $\left\{O_{C h}\right\}$. Thus, we use the second model to identify the moving objects, $\left\{O_{M o}\right\}$ among the objects extracted from the first model that identifies the objects above the road, or 'vertical' objects $\left\{O_{V e}\right\}:\left\{O_{M o}\right\}=\left\{O_{C h}\right\} \cap\left\{O_{V e}\right\},\left\{O_{S t}\right\}=\overline{\left\{O_{C h}\right\}} \cap\left\{O_{V e}\right\}$ and false positives $\{F P\}=\left\{O_{C h}\right\} \cap \overline{\left\{O_{V e}\right\}}$. Note that, here, we manipulate objects rather than windows. These regions are simply defined as sets of neighbor windows.

Figure 5 shows an example of classification result. To estimate $\left\{O_{C h}\right\}$, we can use either radiometric images, or disparity ones. The results for this alternative are very close (cf. Figure 5). In both cases, the houses and pedestrians are classified as static objects. The white car driving in the opposite direction of the camera is classified as a moving object. The gray car driving in the same direction and at similar speed as the camera, is misclassified as a static object, because it is not detected among $\left\{O_{C h}\right\}$ objects, whatsoever using disparity or using radiometry.

Finally let us present the results obtained considering the entire sequence 
of simulated data. When the number of true negatives is undefined, classical indicators are the sensitivity, $S_{e}=\frac{T P}{(T P+F N)}$, the Positive Predictive Value, $P_{P V}=\frac{T P}{(T P+F P)}$, with $T P$ the number of true positives, $F P$ the number of false positives and $F N$ the number of false negatives.

Our ground truth is known at the level of bounding boxes of objects. Therefore, in order not to penalize our results where objects are only partially represented by groups of neighbor windows, TP, FP and $F N$ are calculated as follows. For each image $I_{t}$ of the sequence, $F N$ is the number of objects in the ground truth projected to $I_{t}$ having an empty intersection with the union of the detected windows in $I_{t}, F P$ is the number of groups of neighbor windows detected on $I_{t}$ having an empty intersection with the union of the ground truth objects projected to $I_{t}$ and $T P$ is the number of groups of neighbor windows detected on $I_{t}$ having a nonempty intersection with the union of the ground truth objects projected on $I_{t}$. Table 2 presents the overall results in terms of $S_{e}$ and $P_{P V}$ versus the data input (radiometric or disparity image for $\left\{O_{C h}\right\}$ estimation) and the detection method (monotemporal or multitemporal). We note that $P_{P V}$ is globally much better than $S_{e}$. Now, $P_{P V}$ measures the probability that a detected object is actually an object, whereas $S_{e}$ measures the probability that an object is actually detected. Thus, a high $P_{P V}$ value corresponds to very few false detections and a high $S_{e}$ value corresponds to very few non-detections. Then, the results are in agreement with the basis of the NFA criterion, namely to control the number of false alarms (expectation of FP).

Besides in our sequence the number of actual object varies. In particular, during the last part of the sequence, the car embedding the camera leaves the town and it remains only one object in the scene: a car oncoming from the background of the scene. This car is first not detected inducing $P_{P V}=0$. and then it is detected but there is also a false alarm inducing $P_{P V}=0.5$ decreasing the mean $P_{P V}$ value. During this part of the sequence the significance is very low but not null. Since we do not want to introduce a parameter, we prefer keep the significance threshold equal to 0 . Concerning the poorer performance in term of $S_{e}$ value, they are due to small object(s) far away in the background of the scene. It is the same problem as in the Figure 3 example, where one car and two pedestrians far away from the camera were not detected, because of their small number of pixels (due to the high distance to the camera). 


\begin{tabular}{|c|c|c|}
\hline model $_{1}=$ blank scene but road & $S_{e}$ & $P_{P V}$ \\
\hline model $_{2}=$ projection at $t$ of & $0.754(2 \mathrm{D})$ & $0.897(2 \mathrm{D})$ \\
radiometric image at $t-1$ & $0.683(3 \mathrm{D})$ & $0.914(3 \mathrm{D})$ \\
\hline model $_{2}=$ projection at $t$ of & $0.755(2 \mathrm{D})$ & $0.892(2 \mathrm{D})$ \\
disparity image at $t-1$ & $0.684(3 \mathrm{D})$ & $0.904(3 \mathrm{D})$ \\
\hline
\end{tabular}

Table 2: Statistics of the performance on the simulated sequence. '3D' is for multitemporal method and ' $2 \mathrm{D}$ ' for monotemporal one.

\section{Conclusion and Perspectives}

This study had two aims. Firstly, we wanted to propose a preattentionnal detection algorithm free of supervised parameters. It is based on a strong property of the Number of False Alarm, namely it is a number, that is to say directly comparable to other NFA values. Then, rather than using a threshold, one can directly search a minimum (in NFA values) or a maximum (in significance values that vary as the opposite of the NFA). For change detection application, we proposed a double criterion based on the magnitude of the observed changes at pixel level and the density of the change at window level. Secondly, we wanted to show the genericity of the approach. The kind of detected objects only depends on the input data, namely the innovation at $t$ and the model at $t$. The a-contrario approach is general in the sense that it detects any part of the innovation in contradiction with the model. When the model is a blank scene but the road, it detects the objects salient relatively to the road plane, when the model is a previous image in the sequence, it detects any own moving object, and when the model is a background image, it detects any change relative to this background.

Future work will deal with the optimization of the whole algorithm, both in term of image preprocessing and postprocessing and in term of code acceleration. First, we will enhance the model at $t$. For instance in the case of a background image and static camera, using background updating techniques or multiple background codebook approach we hope removing some false alarms. We will also test our approach on actual data. Second, we wish to use our algorithms in real time on embedded systems for autonomous vehicle. The only way is to reshape and parallelize them, despite their strong serial behavior. According to preliminary tests, we may hope a speedup factor up to 100 on a current GPU Keppler architecture. 


\section{Acknowledgments}

This work was partially funded by a grant from DIGITEO (COMFUCET project).

\section{References}

[1] A. Almansa, A. Desolneux, S. Vamech, Vanishing point detection without any a priori information, IEEE Transactions on Pattern Anaysis Machine Intelligence, $\mathbf{2 5}$ (2003), 502-507, DOI:10.1109/TPAMI.2003.1190575

[2] F. Cao, J. Delon, A. Desolneux, P. Musé, F. Sur, A unified framework for detecting groups and application to shape recognition, Journal of Mathematical Imaging and Vision, 27 (2007), 91-119, DOI:10.1007/s10851-0069176-0

[3] R. Cucchiara, C. Grana, M. Piccardi, A. Prati, Detecting moving objects, ghosts, and shadows in video streams, IEEE Transactions on Pattern Anaysis Machine Intelligence, 25 (2003), 1337-1342, DOI:10.1109/TPAMI.2003.1233909

[4] A. Desolneux, L. Moisan, J.-M. Morel, Meaningful alignments, International Journal of Computer Vision, 40 (2000), 7-23, DOI:10.1023/A:1026593302236

[5] A. Desolneux, L. Moisan, J.-M. Morel, Edge detection by Helmholtz principle, Journal Mathematical Imaging and Vision, 14 (2001), 271-284, DOI:10.1023/A:1011290230196

[6] A. Desolneux, L. Moisan, J.-M. Morel, A grouping principle and four applications, IEEE Transactions on Pattern Analysis and Machine Intelligence, 25 (2003), 508-513, DOI:10.1109/TPAMI.2003.1190576

[7] A. Desolneux, L. Moisan, J.-M. Morel, Gestalt Theory and Computer Vision, In: Seeing, Thinking and Knowing, 38 (2004), 71-101, DOI:10.1007/1-4020-2081-3_4

[8] A. Desolneux, L. Moisan, J.-M. Morel, From Gestalt Theory to Image Analysis: a probabilistic approach, Springer (2008), 276 p, ISBN 978-0$387-74378-3$ 
[9] F. Dibos, S. Pelletier, and G. Koepfler, Real-time segmentation of moving objects in a video sequence by a contrario detection, In: Proceedings of the IEEE International Conference on Image Processing (ICIP'05), 1 (2005), 1065-1068, DOI:10.1109/ICIP.2005.1529938

[10] A. Elgammal, D. Harwood, L. Davis, Non-parametric model for background substraction, In: Proceedings of the 6th European Conference on Computer Vision-Part II (ECCV'00), (2000), 751-767, ISBN:3-540-676864

[11] I. Haritaoglu, D. Harwood, L.S. Davis, W4: real-time surveillance of people and their activities, IEEE Transactions on Pattern Analysis and Machine Intelligence, 22 (2000), 809-830, DOI:10.1109/34.868683.

[12] K. Kyungnam, T.H. Chalidabhongse, D. Harwood, L. Davis, Real-time foreground-background segmentation using codebook model, Real-Time Imaging, 11 (2005), 172-185, DOI:10.1016/j.rti.2004.12.004

[13] R. Labayrade, D. Aubert, J.-P. Tarel, Real time obstacle detection on non flat road geometry through 'V-disparity' representation, In: Proceedings of the IEEE Intelligent Vehicle Symposium'02, 2 (2002), 646-651, DOI:10.1109/IVS.2002.1188024

[14] A. Manzanera, J.C. Richefeu, A new motion detection algorithm based on [Sigma]-[Delta] background estimation, Pattern Recognition Letters, $\mathbf{2 8}$ (2007), 320-328, DOI:10.1016/j.patrec.2006.04.007

[15] L. Moisan, B. Stival, A probabilistic criterion to detect rigid point matches between two images and estimate the fundamental matrix, International Journal of Computer Vision, 57 (2004), 201-218, DOI:10.1023/B:VISI.0000013094.38752.54

[16] F. Porikli, O. Tuzel, Bayesian background modeling for foreground detection, In: Proceedings of the third ACM international workshop on Video surveillance \& sensor networks, (2005), 55-58, DOI:10.1145/1099396.1099407

[17] J. Rabin, J. Delon, Y. Gousseau, A statistical approach to the matching of local features, SIAM Journal on Imaging Sciences, 2 (2009), 931-958, DOI:10.1137/090751359

[18] A. Robin, L. Moisan, S. Le Hégarat-Mascle, An A-Contrario approach for subpixel change detection in satellite imagery, IEEE Transactions 
on Pattern Analysis and Machine Intelligence, 32 (2010), 1977-1993, DOI:10.1109/TPAMI.2010.37

[19] C. Stauffer, W.E.L. Grimson, Adaptive background mixture models for real-time tracking, In: Proceedings of the IEEE Computer Society Conference on Computer Vision and Pattern Recognition, 2 (1999), 2246-2252, DOI:10.1109/CVPR.1999.784637

[20] R.G. von Gioi, J. Jakubowicz, J.-M. Morel, G. Randall, LSD: A fast line segment detector with a false detection control, IEEE Transactions on Pattern Analysis and Machine Intelligence, 32 (2010), 722-732, DOI:10.1109/TPAMI.2008.300

[21] M. Wertheimer, Untersuchungen zur lehre von der gestalt. II, Psychologische Forschung, 4 (1923), 301-350, DOI:10.1007/BF00410640

[22] C.R. Wren, A. Azarbayejani, T. Darrell, A.P. Pentland, Pfinder: real-time tracking of the human body, IEEE Transactions on Pattern Analysis and Machine Intelligence, 19 (1997), 780-785, DOI:10.1109/34.598236 
